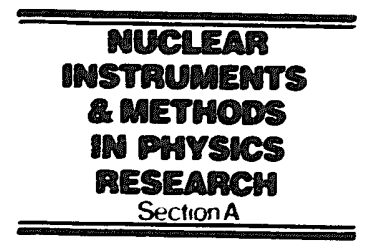

\title{
A compact design for beam transport *
}

\author{
Rainier F.X.A.M. Mols and Gerard J. Ernst \\ Unitersity of Twente, P.O. Box 217, 7500 AE Enschede. The Netherlands
}

\author{
Jan I.M. Botman \\ Eindhoc en University of Technology, P.O. Box 513, 5600 MB Eindhoven, The Netherlands
}

\begin{abstract}
A double-achromatic, double-focusing magnet system of very small size is described which will be used in the injection line for an undulator in a FEL experiment. Because of the double focusing no quadrupoles are needed between the dipole, which couples the electrons in the FEL cavity, and the undulator. An example will be worked out for an electron bunch of $25 \mathrm{MeV}, 100 \mathrm{~A}$ which is focused on a spot of $1 \mathrm{~mm}$ diameter.
\end{abstract}

\section{Introduction}

In the second stage of the TEUFEL project [1] a 25 $\mathrm{MeV}$ electron beam is produced by a photocathode driven microtron. This beam will occupy a transverse phase-space of less than $4 \pi \mathrm{mm}$ mrad and will consist of $30 \mathrm{ps}$ micropulses with a repetition rate of 81.25 $\mathrm{MHz}$. The macropulse duration is $10 \mu \mathrm{s}$.

A transport line has to be used to guide the electrons from the accelerator into the undulator which is placed in an optical cavity. In order to preserve the quality of the beam this system has to be double achromatic and isochronous. The requirement of achromaticity is the easiest to fulfill because the longitudinal properties are related to the micropulse duration: the lengthening of a micropulse should be much smaller than the pulse length in order to keep the high peak current (50-100 A). The influence of the jitter of the micropulses due to the energy spread of $0.5 \%$ is also much smaller than the micropulse duration and therefore negligible.

Our main concern is the geometrical limitation because we want only one micropuise in the optical cavity. Therefore the length of this cavity is $1.846 \mathrm{~m}$. Most of the available space is taken by the undulator of $1 \mathrm{~m}$ length (40 period of $25 \mathrm{~mm}$ ) [2]. The space which is left is used for an incoupling dipole, an extraction dipole and some monitoring devices. We do not have any space left for a focusing quadrupoic

* Sponsored by Nederlands Centrum voor Laser Research (NCLR), the Dutch Foundation for Technological Research (STW) and the Dutch Foundation for Research on Matter (FOM). doublet or triplet in front of the undulator. Therefore, the focusing properties of the beamline have to be correct: the waist has to have a radius of $0.53 \mathrm{~mm}$ at the entrance of the undulator. A last remark is that the distance between the incoupling dipole and the undulator has to be approximately $30 \mathrm{~cm}$ because of a beam positioning monitor in front of the undulator.

On the other hand, the dipoles are restricted to a radius $\rho=12 \mathrm{~cm}$ for a magnetic field $B=0.7 \mathrm{~T}$. Smaller dipoles give higher fields which may saturate and smaller pole faces cause an inhomogeneous field for our gap distance of $2 \mathrm{~cm}$.

\section{System design}

Usually achromatic beam line consists of at least two dipoles. One configuration has to equal bends in the opposite direction and, in between, a quadrupole triplet. This system may have symmetry in the second quadrupole, e.g. as used in the FELIX project [3].

Another simple achromatic system consists of two equal dipoles which bend in the same direction with a quadrupole located symmetrically in between them. Such a system is used at the Los Alamos HIBAF facility [4].

These systems have fixed focusing properties. A set of quadrupoles is used to fit the beam to the undulator. We have included the main focusing action required for a spot of $1 \mathrm{~mm}$ diameter at the entrance of the undulator in a small, doubly achromatic beam system. For this we adapted the second type of beamline mentioned above.

The first step is to replace the quadrupole by a dipole which gives more correction possibilities. The 


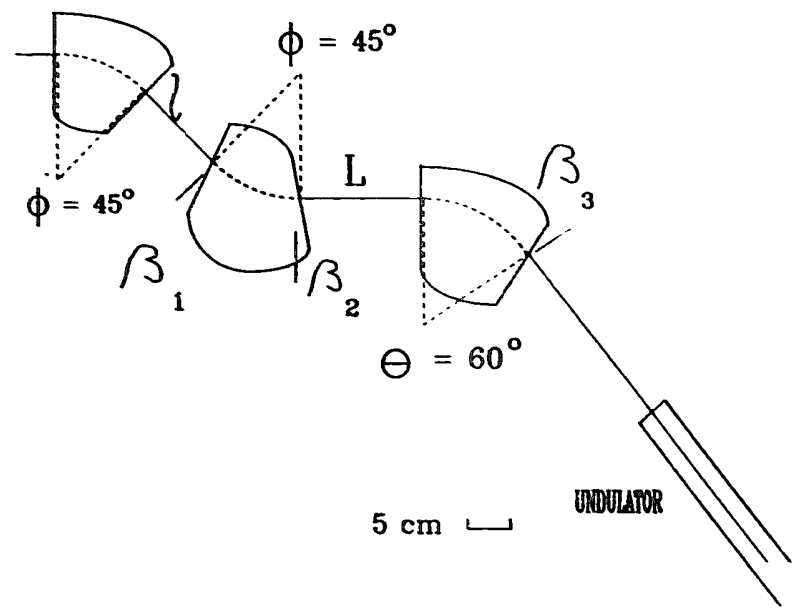

Fig. 1. The transport system consisting of three dipoles. The first two bend $45^{\circ}$ and the incoupling dipole bends $60^{\circ}$. The tilting of the pole faces and the radius are also indicated.

angle is chosen equal to the other dipoles for simplicity. If the bending angle of the last dipole is increased, focusing normal to the bending plane occurs. The double achromaticity is then corrected by changing the distances between the dipoles. Because of this the symmetry properties are lost. The conditions for the double achromaticity are given by (see fig. 1):

$$
\begin{aligned}
l \sin ^{2} \phi= & \rho[\sin \theta-2 \sin \phi(1-\cos \phi)] . \\
L \sin \theta= & \rho[2 \cos \phi(1-\cos \phi)+\cos \theta-1] \\
& +l \cos \phi \sin \phi .
\end{aligned}
$$

where $\rho$ is the dipole radius, $l$ and $L$ are the distances between the dipoles, $\phi$ is the bending angle of the first two dipoles and $\theta$ the bending angle of the last dipole. $\boldsymbol{\theta}>\boldsymbol{\phi}$.

Axial focusing is obtained by tilting the face angles of the magnets. This changes the conditions as mentioned above. The tilting angles are $\beta_{1}, \beta_{2}$ and $\beta_{3}$, where $\beta_{1}$ and $\beta_{2}$ refer respectively to the entrance and exist of the second dipole, and $\beta_{3}$ to the exit of the incoupling dipole. This last angle actually gives the focusing action. Therefore, this angle is approximated by $f=\rho / \tan \beta_{3}$ resulting in $\beta_{3} \simeq 22^{\circ}$.

A similar system as described here is used in radiation therapy linear accelerators [5].

\section{Calculations}

The beam line which is just described has been optimised using the transport codes TRANSPORT [6] and TRANSOPTR [7]. Only the dimensions of the dipoles are fixed: $\phi=45^{\circ}, \theta=60^{\circ}$ and $\rho=12 \mathrm{~cm}$. The starting values for the optimization are given by the above expressions which result in the final solution: $l=13.65 \mathrm{~cm}, L=12.08 \mathrm{~cm}, \beta_{1}=26.6^{\circ}, \beta_{2}=-11.2^{\circ}$ and $\beta_{3}=23.9^{\circ}$. We see from this that the distances between the dipoles only slightly changes and that $\beta_{3}$ is close to the expected value. The initial beam has to have a waist with a radius of $4 \mathrm{~mm}$ at the entrance of the first dipole. The whole system can be described by the following matrix expression:

$\boldsymbol{X}_{\mathrm{f}}=\boldsymbol{R} \cdot \boldsymbol{X}_{\mathrm{i}}$,

where $X$ is a vector describing the properties of the electron beam in the basis $\left(x, \theta_{1}, y, \theta_{1}, \lambda, \delta\right)$ where:

$x: \quad$ the horizontal envelope $(\mathrm{mm})$;

$\theta_{1}$ : the angle between the envelope and the central trajectory (mrad);

$y, \theta_{y}:$ the same as $x$ and $\theta_{\lambda}$ in the vertical plane:

$\lambda: \quad$ half length of the micropulse $(\mathrm{mm})$

$\delta$ : momentum spread $(\%)$.

The initial beam is $\boldsymbol{X}_{\mathrm{i}}=(4,1,4,1,4.5,0.5)$ and $\boldsymbol{R}$ is the first order transport matrix:

$\mathbf{R}=\left[\begin{array}{cccccl}-0.025 & -0.374 & 0 & 0 & 0 & 0 \\ 2.567 & -1.462 & 0 & 0 & 0 & 0 \\ 0 & 0 & -0.007 & 0.370 & 0 & 0 \\ 0 & 0 & -2.693 & -0.424 & 0 & 0 \\ 0 & 0 & 0 & 0 & 1 & 0.097 \\ 0 & 0 & 0 & 0 & 0 & 1\end{array}\right]$.

leading to the desired beam represented by $\boldsymbol{X}_{\mathrm{f}}=$ $(0.53,11,0.53,11,4.54,0.5)$.

In fact, we also added some sextupole focusing to eliminate the aberrations of the dipoles. These corrections were applied to the same surfaces as the tilting, resulting in $R_{1}=-18 \mathrm{~cm}, R_{2}=-10 \mathrm{~cm}$ and $R_{3}=26$ $\mathrm{cm}$ (TRANSPORT notation [6]).

\section{Conclusion}

We have described a doubly achromatic system for a FEL in which focusing properties are included. The isochronous properties are quite good as well: about $600 \mathrm{fs}$ per percent energy spread which will reduce the peak current only about $0.9 \%$ per percent energy spread. Furthermore, there is a practical advantage when actually building such a small system: the dipoles can be mounted on a single base plate with dimensions $50 \times 30 \mathrm{~cm}$ and therefore the total magnetic field of this system can be measured at one time with the correct distances between the dipoles. This makes it casy to tune the correction fields by calculating the orbits of the electrons through the measured field.

\section{References}

[1] G.J. Ernst et al.. these Proceedings (13th Int. Free Electron Laser Conf., Sante Fe. USA. 1991) Nucl. Instr. and Meth. A.318 (1992) 173. 
[2] J.W.J. Verschuur, G.J. Ernst and W.J. Witteman, these Proceedings (13th Int. Free Electron Laser Conf.. Sante Fe, USA, 1991) Nucl. Instr. and Meth. A318 (1992) 847.

[3] R.W.B. Best et al., Technical Report 88-180 Rijnhuizen (1988).

[4] B.E. Carlsten, L.M. Young, M.E. Jones, B. Blind, E.M. Svaton, K.C. Dominic Chan and L.E. Thode, Nucl. Instr. and Meth. A296 (1990) 687.
[5] J.I.M. Botman, T. Bates and H.L. Hagedoorn. Nucl. Instr. and Meth. B10/11 (1985) 796.

[6] K.L. Brown. F. Rothacker. D.C. Carey and Ch. Iselin. CERN 80-(04 (1980)).

[7] E.A. Heighway and M.S. de Jong. AECL-6975. Ontario. Canada (1984). 